\title{
Prospectiva Transdisciplinaria en Educación Superior: Construcción de Perfiles Vocacionales según Grados de Flexibilidad Disciplinar en Estudiantes de Último Año de Nivel Secundario
}

\author{
Tomás Koch(1), Iván Oliva(2), Samuel Valencia ${ }^{(3)}$, Juan P. Salazar(4) \\ (1) Universidad de Playa Ancha, Facultad de Ciencias Sociales. Avda. Playa Ancha 850, \\ Valparaíso-Chile. (e-mail: tomas.koch@upla.cl) \\ (2) Universidad Austral de Chile, Facultad de Filosofía y Humanidades. Campus Isla Teja S/N, \\ Valdivia-Chile. (e-mail: ivanoliva@uach.cl) \\ (3) Gobernación Provincial de Marga Marga, Ministerio del Interior y Seguridad Pública. \\ Manuel Rodríguez 800 Quilpué-Chile. (e-mail: svalenciapfaff@gmail.com) \\ (4) Universidad Austral de Chile, Facultad de Ciencias de la Ingeniería. General Lagos 2086, \\ Valdivia-Chile. (e-mail: juansalazar@uach.cl)
}

Recibido Nov. 7, 2014; Aceptado Dic. 23, 2014; Versión final recibida Ene. 16, 2015

\begin{abstract}
Resumen
En este artículo se analizan los perfiles vocacionales de estudiantes de último año de educación secundaria. Para esto se analizan los resultados de un estudio en una muestra de 1234 jóvenes con los cuales se simuló una postulación a la educación superior. A la vez, se midió la evaluación retrospectiva que estos estudiantes realizan de su formación y la proyección que realizan de la educación superior en términos de flexibilidad y apertura disciplinar. Estos resultados son organizados mediante un análisis de conglomerados en cinco grupos, entre los que destacan conglomerados asociados a la flexibilidad disciplinar, superando clasificaciones vinculadas al clásico binomio decisión/indecisión. Se observa entonces que el problema vocacional puede no encontrarse en el estudiante y su proceso decisional, sino que respondería a una oferta limitada. Esto plantea nuevos desafíos a la organización del currículo en educación superior.
\end{abstract}

Palabras clave: estudiantes secundarios, perfil vocacional, transdisciplinariedad, relación escuelauniversidad

\section{Transdisciplinary Prospective in Higher Education: Construction of Vocational Profiles of Senior High School Students according to their Disciplinary Flexibility Degrees}

\begin{abstract}
This article analyses vocational profiles of senior high school students. A sample of 1234 students was considered simulating the application process to enter higher education. Through a simulated application, participants were asked to both evaluate the education they received and make choices on the prospective training they would seek at university level. With this we aimed at gaining insight into disciplinary openness and flexibility. The results were organized into five groups obtained through cluster analysis. Among these, clusters associated to disciplinary flexibility overcome the classic binary decision/indecision understanding of career choice. Therefore, it is concluded that the problem of career choice may not be intrinsic to students and their decisional process, but rather to a limited offer of study programs. This highlights new challenges for curriculum planning in higher education
\end{abstract}

Keywords: secondary students, vocational profile, transdisciplinarity, school-university transition 


\section{INTRODUCCIÓN}

La composición de la matrícula en educación terciaria en Chile, se ha modificado significativamente producto de la masificación del acceso a este nivel; no obstante, la oferta educativa de las universidades en términos cualitativos, continúa siendo predominantemente rígida en términos disciplinares y profesionales. Ello condiciona la emergencia de modelos de educación más flexibles e inter-transdiciplinarios, relevando la importancia de comprender los procesos y perfiles vocacionales de los estudiantes que postulan a las universidades. Desde esta base, la investigación se orientó a comprender las manifestaciones del imaginario referido a la organización disciplinar en el tránsito desde la educación secundaria a la educación superior.

En este contexto, la predominante especialización curricular y las hegemónicas formas de organización disciplinar en la educación superior en Chile, configuran a nuestro juicio, un nodo crítico para el desarrollo cualitativo de sus procesos formativos. Derivado de lo anterior, sostenemos que una deficiencia estructural de los modelos curriculares y la política de educación terciaria en este contexto, está asociada a una flexibilidad curricular limitada, sobre-especialización del curriculum y sistemas de conocimiento disciplinares autorreferenciados. Asimismo, la evaluación de la pertinencia y sustentabilidad de programas inter y transdisciplinarios más flexibles, requieren perspectivas de observación que atiendan a sus principales interfaces contextuales con el medio escolar.

El fenómeno de la elección de área disciplinar para la continuidad de estudios ha sido un tema ampliamente abordado principalmente desde perspectivas psicológicas, las que tradicionalmente lo han leído desde el binomio decisión/indecisión. Estos estudios han reconocido múltiples formas de indecisión (Germeijs \& De Boeck, 2003; Santos et al., 2014); no obstante, todos ellos asumen la necesidad de elección en base a una oferta curricular disponible, situando el problema decisional a nivel del estudiante (Gati et al., 1996). De este modo, dicho énfasis se encuentra vinculado al esquema organizacional de la sociedad en la que se enmarca el proceso decisional y al sentido subsidiario atribuido a la educación respecto a otros sistemas en estos contextos.

Nuestra perspectiva se distancia de estas concepciones en tanto vincula los procesos de construcción de perfiles vocacionales con el modo de organización disciplinar de la educación y el sentido de la misma en el contexto social. Para esto, caracterizamos los perfiles vocacionales de estudiantes de último año de educación secundaria, atendiendo a sus grados de flexibilidad vocacional y su correlato con las lógicas curriculares predominantes de la educación terciaria chilena y las emergentes perspectivas de desarrollo inter-transdisciplinarias en sus procesos formativos. Desde esta base, se generó una caracterización de los perfiles vocacionales de los/as estudiantes, atendiendo a los grados de apertura/clausura disciplinar observados.

La educación ha estado siempre asociada a distintos sistemas sociales, adoptando la función de socialización para la vida en sociedad. Una de las relaciones de más larga data, es la que existe entre educación y sistemas productivos. Las descripciones de Durkheim a principios de 1900 ya sindicaban a la educación como un mecanismo vinculado a las necesidades sociales y de este modo a la división social del trabajo (Durkheim 2012). En este contexto, la tendencia hacia la especialización se presenta como producto del desarrollo de las sociedades. La relevancia asignada a la división del trabajo y especialización aparece con fuerza en la obra de Adam Smith, quien dedica los primeros tres capítulos de su obra "La Riqueza de la Naciones" para trabajar el concepto de la especialización y comienza su primer capítulo señalando que "EI progreso más importante en las facultades productivas del trabajo, y gran parte de la aptitud, destreza y sensatez con que éste se aplica o dirige, por doquier, parecen ser consecuencia de la división del trabajo" (Smith, 2011), situando con ello, a la especialización en el centro de la teoría del crecimiento económico.

A partir de la construcción teórica de Adam Smith, se entiende la división del trabajo como uno de los pilares fundamentales del paradigma de producción fordista (Boyer y Freyssenet 2002; Jiménez, 2009; Arvidson, 2011). Donde las características de este modelo, sobre todo las relacionadas a la atomización de los procesos y la estandarización de la tareas de producción resultan significativas y reveladoras para comprender la importancia de la especialización y la división del trabajo en la producción de masas como un recurso para aumentar la productividad y el desarrollo económico de los países en las primeras décadas del siglo XX. En la década de 1970 y en el marco de una gran crisis capitalista a nivel mundial, el sistema productivo fordista da cuenta de su agotamiento, volcándose las miradas de la producción industrial hacia otros modelos, como el japonés, el cual permitió un acelerado desarrollo de su industria. Las características de este modelo de producción se asocian a la flexibilidad laboral y alta rotación en los puestos de trabajo/roles y estímulos sociales a través del fomento del trabajo en equipo y la identificación transclase entre jefe-subalterno (Coriat 1992; Freyssenet 2011). 
Las actuales transformaciones de las economías globales basadas en el conocimiento, demuestran la incongruencia entre la hiperespecialización y los nuevos modelos productivos postfordistas en sus distintas hibridaciones (Mehralizadeh et al., 2008). Bajo estos nuevos parámetros, se descompone la relación desarrollo económico - división del trabajo, debido a que los nuevos modelos flexibles de producción (y contratación) requieren de trabajadores flexibles, que conozcan el producto que generan en su amplitud y que puedan realizar distintas tareas productivas, volviendo relevante la pregunta por el perfil profesional que fomentan las instituciones educativas (Campion \& Renner, 1992).

\section{Educación superior en Chile: acceso y características}

En las últimas dos décadas, la educación superior chilena ha experimentado cambios sustantivos, sobre la base del crecimiento exponencial de la matrícula en educación terciaria. Este crecimiento se ha visto plasmado en una mayor oferta de universidades y programas. La cobertura en educación superior aumentó de 520.000 estudiantes en 2002 a 1.100 .000 en 2012. Según OCDE y BM (2013), en 2010 el $71 \%$ de la población entre 25 y 64 años había alcanzado al menos la educación secundaria en Chile. De la misma manera, el 38\% de los jóvenes entre 25 y 34 años alcanzó la educación superior. En el año 2010 , el porcentaje total de graduación en Chile fue cercano al 20\% para los programas de educación superior. Respecto al área de estudios, el 2011, el área con mayor tasa de matriculación fue la de Tecnología $26 \%$, seguida por los programas de Ciencias de la Salud con un 19\%, y en tercer lugar Ciencias Sociales y Pedagogía con un 14\%. Este progresivo aumento de la matrícula de la educación superior chilena ha modificado el perfil de ingreso de los estudiantes de este nivel educativo, masificándose el acceso (Larrain y Zurita, 2008). Este incremento de la cobertura educacional, se refleja en una mayor oferta de instituciones de educación terciaria, existiendo en Chile actualmente 178 Instituciones de educación superior con reconocimiento oficial, en donde se imparten más de 2100 programas universitarios distintos (Consejo Nacional de Educación CNED, 2013).

El financiamiento de la educación de estas nuevas masas de estudiantes, se basa actualmente en un sistema de financiamiento privado, donde el rol del Estado se traduce en un financiamiento a la demanda, a través de un sistema de becas y créditos (Larrain y Zurita 2008). En cuanto a las primeras, éstas están enfocadas principalmente a los quintiles de ingresos más bajos, mientras que los segundos, son el mecanismo de mayor masividad, siendo dos los principales: el Fondo Solidario de Crédito Universitario (FSCU) -enfocado solo a ciertas universidades y con restricciones de tipo socioeconómico- y el Crédito con Aval del Estado (CAE) -crédito intermediado por la banca-. Este sistema de financiamiento ha sido foco de cuestionamiento por parte de la sociedad chilena en tanto transfiere a las familias el costo de la educación superior, logrando la masividad a través del endeudamiento (Salinas y Fraser, 2012). Más allá del financiamiento y el aumento cuantitativo en el acceso a la educación terciaria en el país, este nuevo marco de la educación superior chilena hace emerger problemáticas cualitativas asociadas a la organización curricular, sus grados de flexibilidad y sus implicancias en las altas tasas de deserción del sistema.

El informe de la OCDE (2009) ha señalado la flexibilidad limitada y la sobre especialización del curriculum como deficiencias estructurales genéricas del sistema, diagnosticando enfoques curriculares generalmente inflexibles y demasiado especializados. En relación a la flexibilidad limitada, se señala el énfasis de un currículo rígido, con opciones muy limitadas o sin asignaturas electivas una vez que el estudiante ha escogido un área de especialización. Esta flexibilidad limitada, además, es uno de los principales impedimentos para la articulación y movilidad entre programas y niveles académicos en Chile (Gaete \& Morales, 2011). En lo referido a la sobre-especialización del currículo, el mismo informe OCDE (2009) señala que la mayor parte de los programas académicos ponen gran énfasis en la preparación para un campo de estudio específico, dando gran énfasis en una variedad de materias orientadas profesionalmente, pero no incluyendo cursos de educación general, destacando que los estudiantes deben elegir un campo académico al inicio de sus estudios, donde salvo pocas excepciones, no se permite la movilidad lateral entre programas académicos, incluso dentro de la misma institución. Este factor, combinado con la limitada orientación vocacional en la enseñanza secundaria (Mc Whirter \& Mc Whirter, 2012) tiene gran influencia en las tasas de deserción en la educación terciaria en el país, situándose junto a la situación económica de la familia y el rendimiento académico, como una de las causas que más influyen en la deserción durante el primer año (Centro de Microdatos de la Universidad de Chile, 2008).

\section{Elección vocacional: apertura y clausura disciplinar/profesional}

Pese a este aumento de la cobertura en educación superior, Chile no ha podido dar respuesta a las altas tasas de deserción en la educación terciaria. Las tasas de deserción al término del primer año universitario, de acuerdo al Consejo Superior de Educación (CSE 2012), son cercanas al 20\% promedio y del orden del $40 \%$ al finalizar el tercer año. La situación se vuelve más crítica aún en la formación Técnica, donde estas instituciones tienen tasas de deserción cercanas al $43 \%$ al finalizar el primer año. 
Sin duda los programas de nivelación y apoyo económico y social son un aporte para combatir la deserción, no obstante, dada la centralidad del factor vocacional en esta materia, resulta necesario abordarlo desde ópticas complementarias. Desde esta base, entendemos que una dimensión importante de la deserción universitaria puede deberse no solo a factores de la efectividad de la elección, sino también a problemáticas de rigidez curricular disonantes a los perfiles vocacionales estudiantiles. En consecuencia, una parte importante de los indicadores de deserción podrían responder no solo a paradigmas remediales y de nivelación, sino más bien a campos de no equivalencia entre las estructuras vocacionales estudiantiles y las lógicas curriculares rígidas de especificidad disciplinar predominantes en la educación terciaria en el país.

Esta situación, nos hace reenfocar la mirada no solo al acceso a la educación superior, sino que plantea nuevos desafíos a fin de comprender los procesos epistemológicos a partir de los cuales se construye la elección vocacional, a la vez de plantear serios cuestionamientos a las estructuras curriculares y sus grados de flexibilidad (Oliva, et al., 2014). Siendo para Chile un desafío crucial la revisión de la pertinencia de sus universidades en el escenario global, se constata que la estructura de la gran mayoría de ellas mantiene una orgánica en términos de facultades y departamentos que refuerza aún más la formación unidisciplinaria (Max-Neef, 2004). Desde esta base, nuestro objetivo fue explorar cómo y dónde se generan los límites disciplinarios/profesionales en la construcción de perfiles vocacionales en jóvenes de último año de educación secundaria, atendiendo a sus implicancias en el fomento de estructuras curriculares más flexibles, complejas e inter-transdisciplinares en la educación superior en el país.

\section{MATERIALES Y MÉTODOS}

Los datos utilizados en este artículo corresponden a una encuesta social aplicada a una muestra de 1234 estudiantes que cursaban el último año de educación secundaria en Chile, el año 2013. La selección de los casos se realizó a través de un muestreo por conglomerados, donde se seleccionaron establecimientos educacionales distribuidos a lo largo del territorio nacional buscando responder a tres macrozonas definidas como Norte (XV, I, II, III y IV Regiones), Centro (V, Metropolitana, VI y VII Regiones) y Sur (VIII, IX, XIV, X, XI y XII Regiones). En términos la composición por género, la muestra presenta cerca de un $51 \%$ de hombres y un $49 \%$ de mujeres y respecto a la dependencia administrativa de los establecimientos seleccionados, cerca de un $11 \%$ corresponde a estudiantes de establecimientos particulares, un $36 \%$ a establecimientos particulares subvencionados y un $52 \%$ a establecimientos municipales.

El instrumento utilizado fue confeccionado expresamente para este estudio, cuenta con 42 items agrupados en cuatro secciones: i) información general tanto del individuo como del establecimiento educacional; ii) simulación de postulación a la educación superior; iii) evaluación de la organización e interacción de las áreas de conocimiento en los 12 años de educación primaria y secundaria obligatoria; y iv) criterios de valoración de la oferta de carreras en cuanto a sus grados de flexibilidad curricular e intertransdisciplinariedad.

A partir de los resultados, se construyeron tres índices: "Índice de flexibilidad en la elección de carrera" (INFLEXED) Calculado a partir de sumatoria de los puntajes asignados de acuerdo al número de dominios disciplinarios (OCDE 2002) a los que simuló postular el estudiante (3 puntos cada uno) y el número de casas de estudio (1 punto cada una); "Índice de evaluación de la organización e integración de las áreas de conocimiento escolares" (INEVORIACE) e "Índice de imaginario de carreras universitarias" (INICU). Tanto el INEVORIACE como el INICU son calculados a partir del puntaje obtenido en escalas de Diferencial Semántico, con 9 y 10 items respectivamente, lo que implica puntajes máximos de 45 y 50 puntos. Ambas escalas resultan confiables, con un Alfa de Cronbach de 0,806 y 0,814 respectivamente.

El primero de estos índices, refiere al grado de flexibilidad que presentan los estudiantes al momento de simular una postulación a diferentes carreras universitarias, en el sentido que mientras más alto el indicador, mayor será grado de flexibilidad; el segundo índice está asociado a la evaluación retroactiva que realizan los estudiantes de su educación primaria y secundaria de acuerdo a su precepción de la integración de los diversos campos disciplinares del currículo escolar, en donde mayor puntaje implica peor evaluación; mientras que el tercero refiere en términos proyectivos, a los grados de flexibilidad e interdisciplinariedad que los estudiantes esperan de las carreras y universidades a las cuales postulan, donde puntajes más altos indicarían imaginarios más flexibles. Estos índices fueron analizados en términos de sus frecuencias en torno a dos variables estructurales, el sexo del estudiante y la dependencia administrativa del establecimiento, la que dado el alto nivel de segregación escolar, funciona en el caso chileno como indicador del nivel socioeconómico de las familias (Valenzuela, et al., 2014). Finalmente, a partir de un análisis de conglomerados, se propone una tipología de perfiles de acuerdo a la combinación de estos tres índices, la que es analizada en términos de las variables estructurales enunciadas (sexo y dependencia del establecimiento). 
La conformación de conglomerados a partir de un análisis de Cluster, permitió clasificar los grados de flexibilidad del perfil vocacional en relación a los rangos de apertura y clausura disciplinar expresados por los jóvenes. En este contexto, una clasificación de los perfiles vocacionales en conglomerados resultaría pertinente para orientar las decisiones de los jóvenes y/o modelar la oferta curricular. Por ejemplo, un perfil vocacional tendiente a la apertura disciplinar tendría mayor posibilidad de éxito y permanencia en una carrera con mayores grados de flexibilidad curricular, mientras que un estudiante con este mismo perfil en una carrera más tendiente a la clausura disciplinar, presentaría más probabilidades de deserción.

\section{RESULTADOS Y ANÁLISIS}

En relación al proceso de elección de carrera, se observó que los estudiantes eligen mayoritariamente entre una y dos carreras (25,9 y $29,3 \%$ respectivamente), solo registrándose un $9,4 \%$ de los casos donde se escogió el máximo número posible de carreras (5). Al calcular la distribución por dominios disciplinarios de acuerdo a la clasificación de la OCDE (2002), se observa que más de la mitad de los estudiantes (53,4\%) postula a carreras pertenecientes a un mismo dominio disciplinario. Estos datos, si bien no son directamente un indicador de la flexibilidad vocacional, nos permiten una primera aproximación en términos proyectivos al perfil vocacional de las estudiantes. Asimismo, un 9,4\% de los casos presentan una elección de 4 o 5 dominios disciplinarios, lo que a priori podría indicar un alto grado de flexibilidad cognitiva, sin embargo, al cruzar estos datos con los otros índices, encontramos que un porcentaje de este grupo no presenta una perspectiva flexible ni en términos retroactivos (INEVORIACE) ni proyectivos (INICU), lo que nos hace hipotetizar que corresponden más bien a lo que la literatura identifica típicamente como "indecisión" (Kelly e Lee, 2002).

Estas características son las que recoge el Índice de Flexibilidad en la Elección de Carrera (INFLEXED), donde se consideraron las postulaciones que los estudiantes simularon de acuerdo a los dominios disciplinares de las carreras y el número de instituciones a las cuales postularon. La figura 1 muestra la distribución de este índice según dependencia administrativa del establecimiento, donde se aprecian diferencias entre las medias de los tres grupos, las que resultan estadísticamente significativas $(p<0,05)$ y que muestran una correlación de este índice con el nivel socioeconómico de los establecimientos, encontrándose en los colegios particulares pagados la media más alta $(7,43)$, mientras que estudiantes de colegios municipalizados obtienen la media más baja $(6,72)$.

Esta relación, a la luz de los resultados de investigaciones anteriores (Oliva, et al., 2014) y siguiendo a Bourdieu y Passeron (2009), es posible de ser interpretada no solo desde el acceso a la información de estos jóvenes, sino que desde el sentido que tiene la educación superior para los estudiantes de distinto nivel socioeconómico, donde en los niveles socioeconómicos más bajos, encontramos en forma mucho más persistente un sentido utilitario del paso por la educación superior, dado que es vista como mecanismo de movilidad social y empleabilidad inmediata (PNUD, 2012). Esto se potencia por el hecho de que en Chile el modelo de financiamiento de la educación superior pone la carga en las familias, por lo que asegurar un retorno económico resulta fundamental para estos jóvenes, alineando sus esfuerzos en esta dirección y reproduciendo una versión elitista de currículos más flexibles e interdisciplinarios.

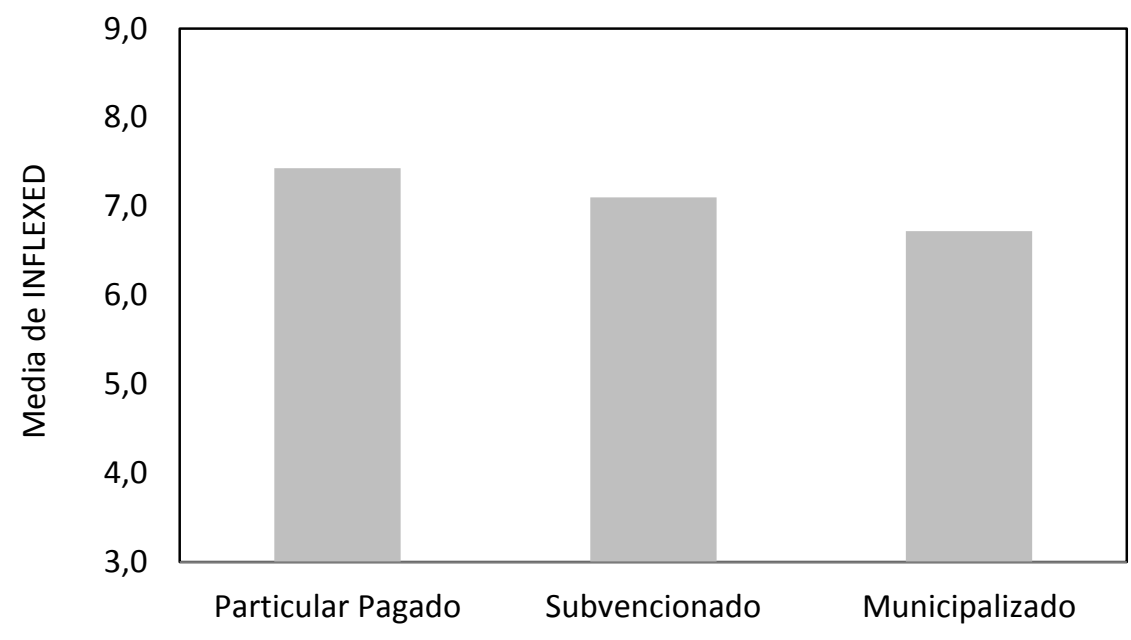

Fig. 1: Distribución INFLEXED según dependencia administrativa 
Al asociar el INFLEXED al sexo de los estudiantes, observamos que las diferencias no resultan estadísticamente significativas, no pudiendo asumirse, al menos a partir de este indicador, diferencias por género asociadas a la flexibilidad vocacional de los estudiantes. En lo que se refiere a la evaluación retrospectiva de su paso por la educación primaria y secundaria, se calculó el Índice de la Organización e Interacción de las Áreas de Conocimiento Escolar (INEVORIACE), el cual puede ser considerado como un indicador de la flexibilidad cognitiva en tanto utiliza este argumento como parámetro para la evaluación de su experiencia escolar.

En general este indicador se comportó normalmente, presentando puntajes que se distribuyen en toda la escala (los puntajes oscilan entre los 9 y 45 puntos, siendo estos valores también los máximos y mínimos teóricos) y presentando una media de 21,4 puntos, situándose por debajo de la media teórica, lo que estaría indicando en general, una percepción relativamente positiva de la organización de las áreas de conocimiento en la educación escolar. Si bien encontramos algunas diferencias cuando contrastamos esta información con la dependencia administrativa del establecimiento, tal como se aprecia en la figura 2 , éstas no resultan significativas $(p>0,05)$. Estas diferencias pueden ser preliminarmente atribuidas a las diferencias objetivas que existen entre estos establecimientos en términos de los conocimientos recibidos, el acceso a la educación superior e infraestructura (OCDE 2009).

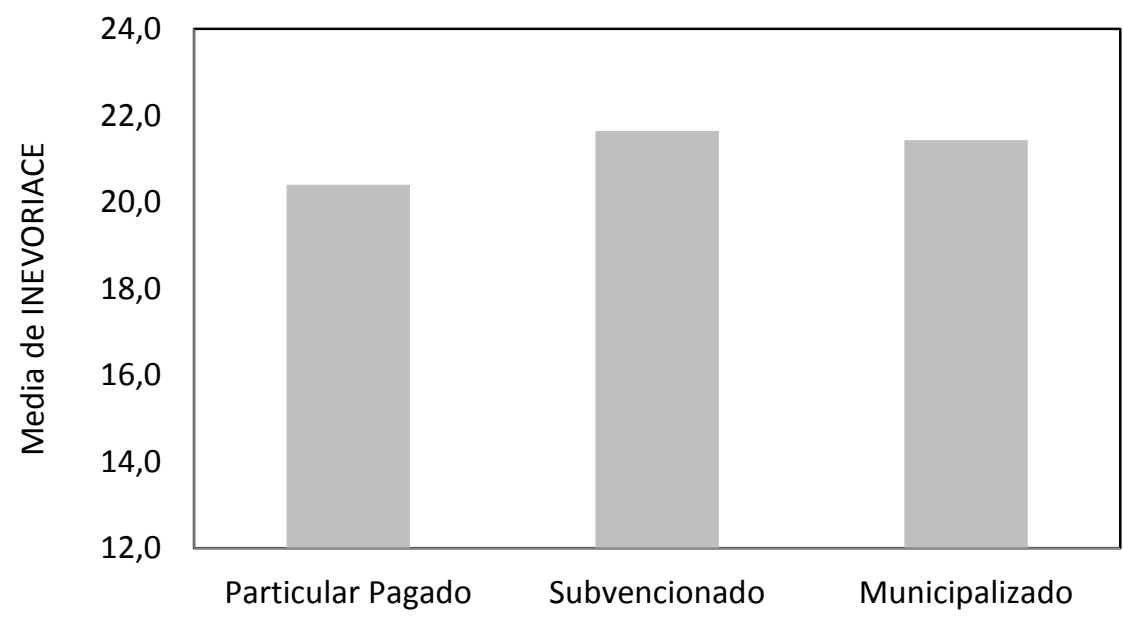

Fig. 2: Distribución INEVORIACE según dependencia administrativa

Si bien en términos generales se aprecia la distinción anteriormente señalada, al observar la distribución de las medias por pregunta (Figura 3), encontramos que las respuestas se mueven en el mismo sentido independiente de la dependencia del establecimiento, presentando similares desviaciones (entre 0,8 y 1,2) siendo en todos los casos, considerada útil y necesaria la educación recibida, lo que habla acerca de la alta valoración social de la educación, siendo las variables estructura, dispersión e independencia de los saberes los ámbitos peor evaluados.

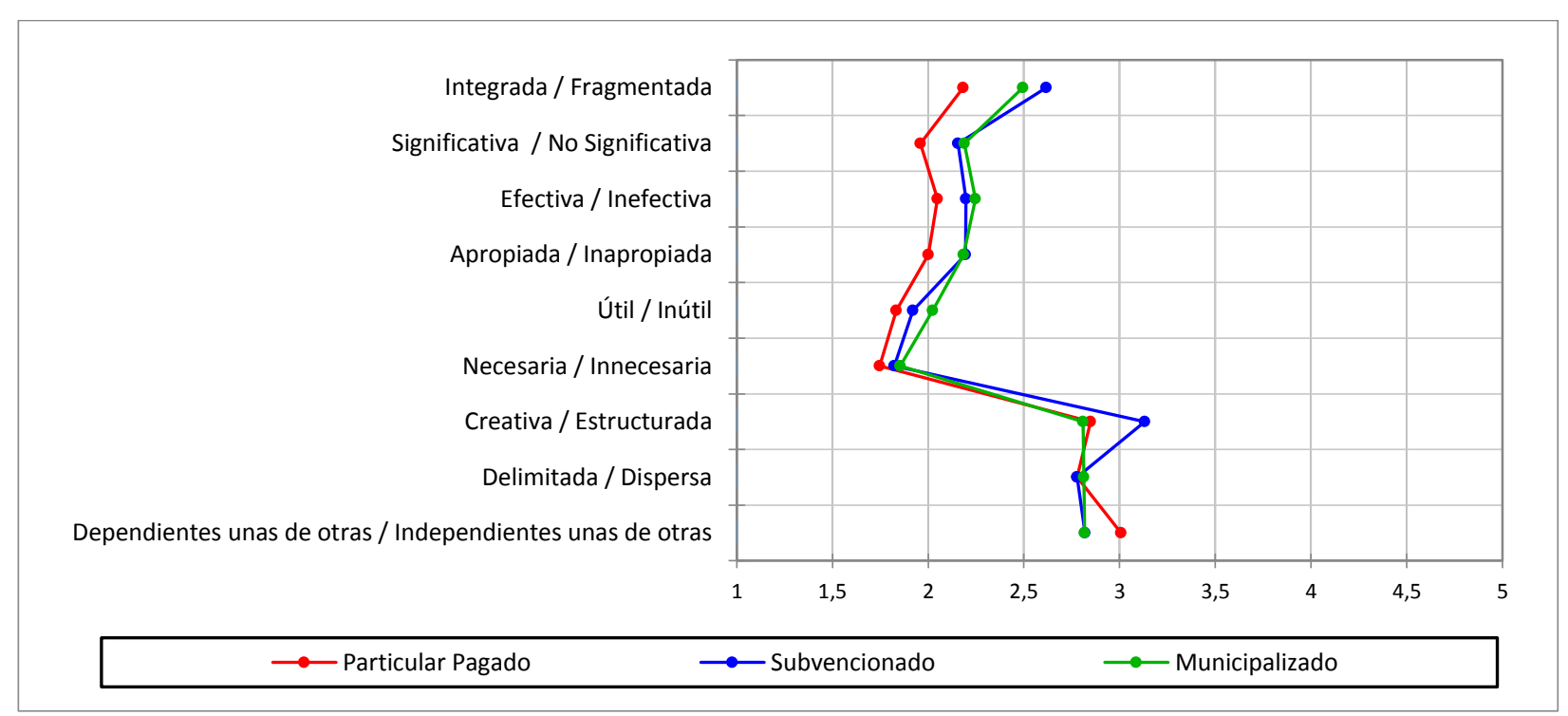

Fig. 3: Distribución de las medias a los ítems de la escala de evaluación de la educación (INEVORIACE) según dependencia administrativa del establecimiento 
A diferencia del caso de la dependencia administrativa, cuando comparamos la evaluación de la educación recibida según la distribución por sexo (Figura 4 ), encontramos diferencias significativas $(p<0,01)$, donde los hombres sistemáticamente realizan una peor evaluación que las mujeres. Esta situación se observa en prácticamente todas las preguntas de la escala. Tal como en el caso anterior, también observamos como todos los ítems se mueven en la misma dirección según sexo y presentan desviaciones similares entre 0,9 y 1,2 .

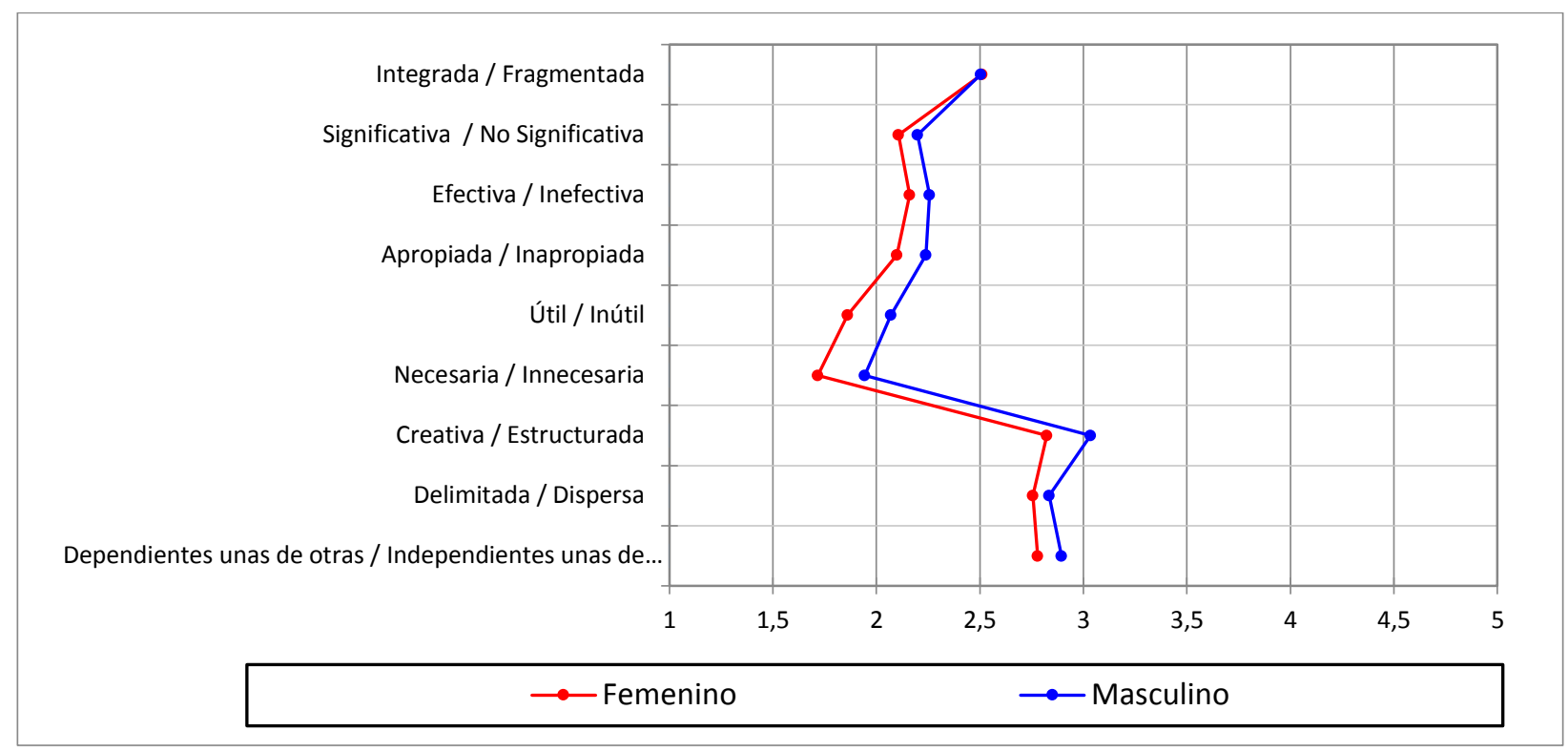

Fig. 4: Distribución de las medias de los ítems referidos a evaluación de la educación (INEVORIACE) según sexo

Finalmente, el tercer elemento que se consideró en la construcción de perfiles vocacionales según su grado de flexibilidad, fue la visión proyectiva que presentan los estudiantes. Esto fue evaluado a través del Índice de Imaginario de Carreras Universitarias (INICU), que funciona como indicador respecto al nivel de flexibilidad que desearían encontrar en la educación universitaria.

Los puntajes de este indicador se distribuyeron normalmente a través de toda la escala, siendo los puntajes máximos y mínimos reales equivalentes a los teóricos (10 y 50 respectivamente) y presentando una media de 24,1 la que está ligeramente por debajo de la media teórica de 25. Presentando una desviación de 7,2.

Esta situación de normalidad cambia cuando realizamos el análisis por dependencia administrativa del establecimiento (Figura 5), puesto que las diferencias que se presentan entre los establecimientos resultan estadísticamente significativas con $p<0,01$ en el sentido de que a medida de que aumenta el nivel socioeconómico aumenta el grado de flexibilidad deseado en la educación superior. Este elemento viene a reafirmar la concepción elitista de un currículo más flexible e interdisciplinario, a través de los elementos ya enunciados, información, condicionantes materiales y motivación para el estudio en la educación superior.

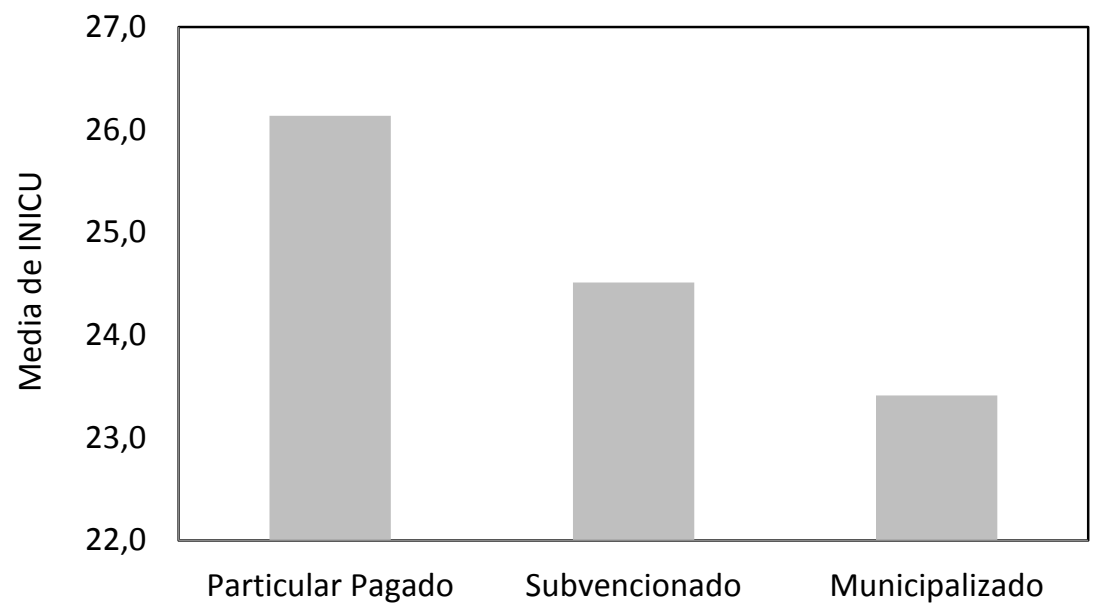

Fig. 5: Distribución del INICU en estudiantes de cuarto medio según dependencia del establecimiento educacional. 
Estas diferencias, sobre todo la diferencia relativa al alto puntaje INICU de los colegios particulares pagados, es explicada principalmente por las respuestas de las mujeres, encontrándose que si bien no existen mayores diferencias del comportamiento del índice según sexo, al controlar la relación de dependencia administrativa por sexo, se aprecia que en el grupo de los establecimientos particulares pagados son las mujeres las que establecen las diferencias, siendo el puntaje de los hombres similar al de los otros grupos. Esta información resulta de interés, y podemos plantear a modo de hipótesis a partir de indagaciones cualitativas, que esta flexibilidad es asociada a la libertad por las mujeres de este segmento, puesto que existe una imagen transmitida desde la familia, que asocia la educación con independencia económica y así con libertad (Koch, Contreras y Oliva 2014; PNUD 2012).

En lo referente al análisis por ítems, tal como se aprecia en la Figura 6, encontramos que la distribución por sexo se mueve en la misma dirección tanto para hombres como para mujeres en todos los ítems, misma situación que sucede con la dependencia del establecimiento, a excepción del ítem "campo laboral fijo / campo laboral flexible", en donde los establecimientos que se asocian a clases más bajas (municipales) se inclinan en mayor medida por un campo laboral fijo, reafirmando los elementos anteriormente señalados respecto al carácter utilitario que tiene para estos jóvenes el acceso a educación superior en tanto mecanismo de movilidad social y de rentabilizar la inversión realizada a través del endeudamiento (créditos para estudiar).

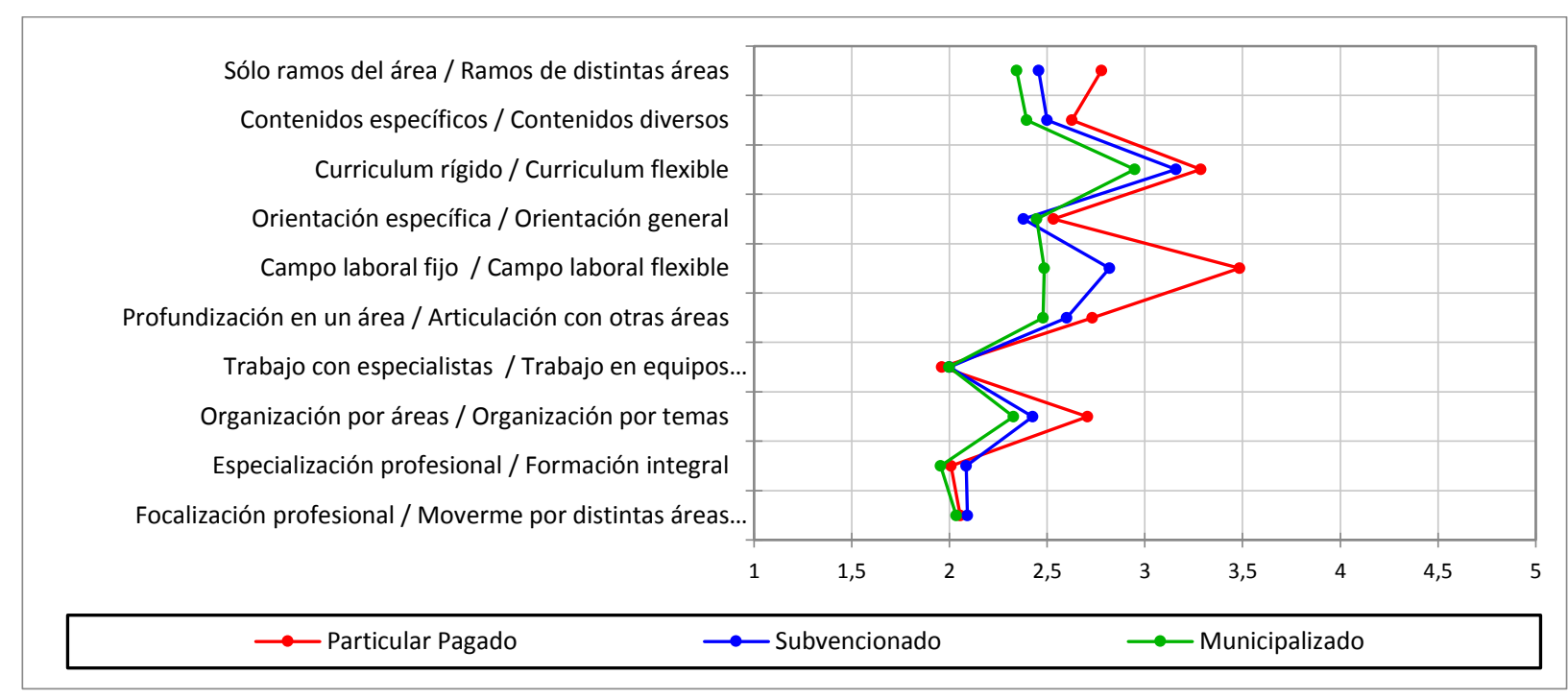

Fig. 6: Distribución de las medias a los ítems de la escala de imaginario de carrera (INICU) según dependencia administrativa del establecimiento

\section{Análisis de conglomerados}

A continuación se presenta una propuesta de clasificación de perfiles vocacionales elaborada a partir de estos tres índices (INFLEXED, INEVORIACE e INICU), donde a través de un análisis de conglomerados se organizan 5 grupos que presentan un nivel aceptable de cohesión interna y de distancia entre ellos. Estos grupos fueron nombrados de acuerdo a las características que presentan sus casos. Es así como estos cinco perfiles son: "Especialistas", "Flexibles", "Especialistas críticos", "Especialistas integrales" e "Indefinidos". La tabla 1 presenta la distribución del número de casos bajo las clasificaciones propuestas.

Tabla 1. Clasificación de los perfiles vocacionales

\begin{tabular}{|c|c|c|}
\hline & Frecuencia & Porcentaje \\
\hline Especialistas & 198 & 16,05 \\
\hline Flexibles & 317 & 25,69 \\
\hline Especialistas integrales & 179 & 14,51 \\
\hline Especialistas críticos & 215 & 17,42 \\
\hline Indefinidos & 325 & 26,34 \\
\hline Total & 1234 & 100 \\
\hline
\end{tabular}


Esta clasificación se distancia de otras asociadas a tipos de habilidades (Thomas y Menz 1996) y sigue la línea de la construcción de perfiles asociando la decisión vocacional a otros elementos (Gamboa, Paixão y Neves 2014), que en este caso refieren a la apertura/flexibilidad con que evalúan su experiencia educativa y el imaginario de carrera que construyen. Si bien la clasificación propuesta es estrictamente dependiente de los datos analizados en tanto técnica descriptiva, se presenta aquí a modo de ejercicio ejemplificador de cómo la categorización de los perfiles vocacionales de los estudiantes puede enriquecerse en tanto se consideran variables asociadas a procesos más complejos que el par decisión/indecisión.

En términos generales, se aprecia que existe una diferencia significativa $(p<0,05)$ en la composición de los cuatro grupos según las variables sexo y dependencia del establecimiento (Figuras 7 y 8). Estas diferencias, en el caso de la dependencia administrativa del establecimiento están principalmente marcadas por las categorías "Flexibles" y "Especialistas integrales", donde los estudiantes de colegios particulares - y en menor medida particular subvencionados- presentan más estudiantes con este perfil. Esta situación viene a reforzar el carácter elitista que presenta hoy en día la inter/transdisciplina y señala una luz de alerta en tanto pudiese estarse convirtiendo en un nuevo mecanismo de diferenciación y desigualdad social.

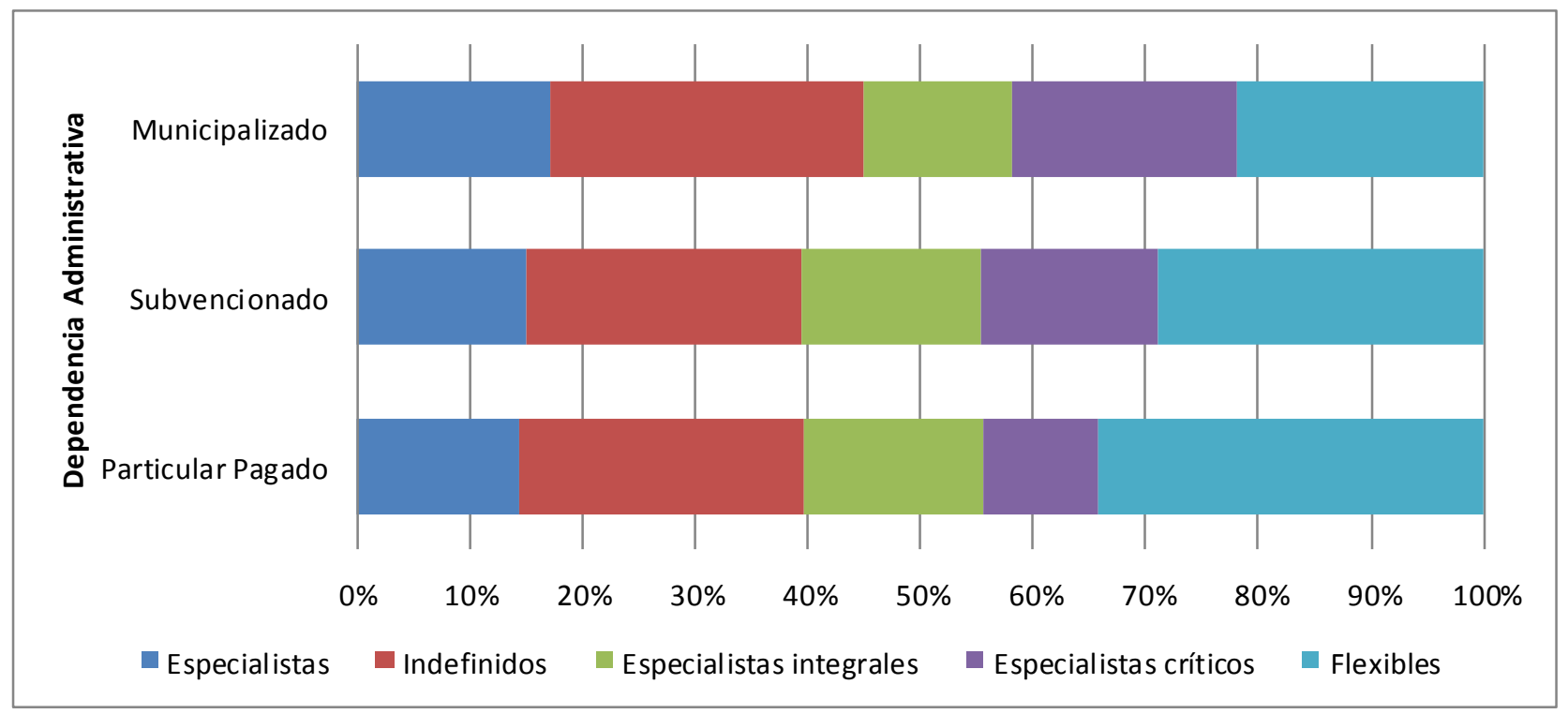

Fig. 7: Establecimiento educacional según pertenencia a conglomerado.

Respecto a las diferencias por sexo, observamos que se dan principalmente en los conglomerados de Especialistas Integrales y Críticos, donde se establece una diferencia a favor de las mujeres en el primer grupo mientras que esta diferencia es a favor de los hombres en el segundo. Esta situación puede ser explicada a partir de los elementos anteriormente señalados en torno a la alta valoración de la educación que presentan las mujeres a la vez que una imagen más bien especializante respecto a la universidad. A continuación se presenta una breve descripción de las tipologías construidas.

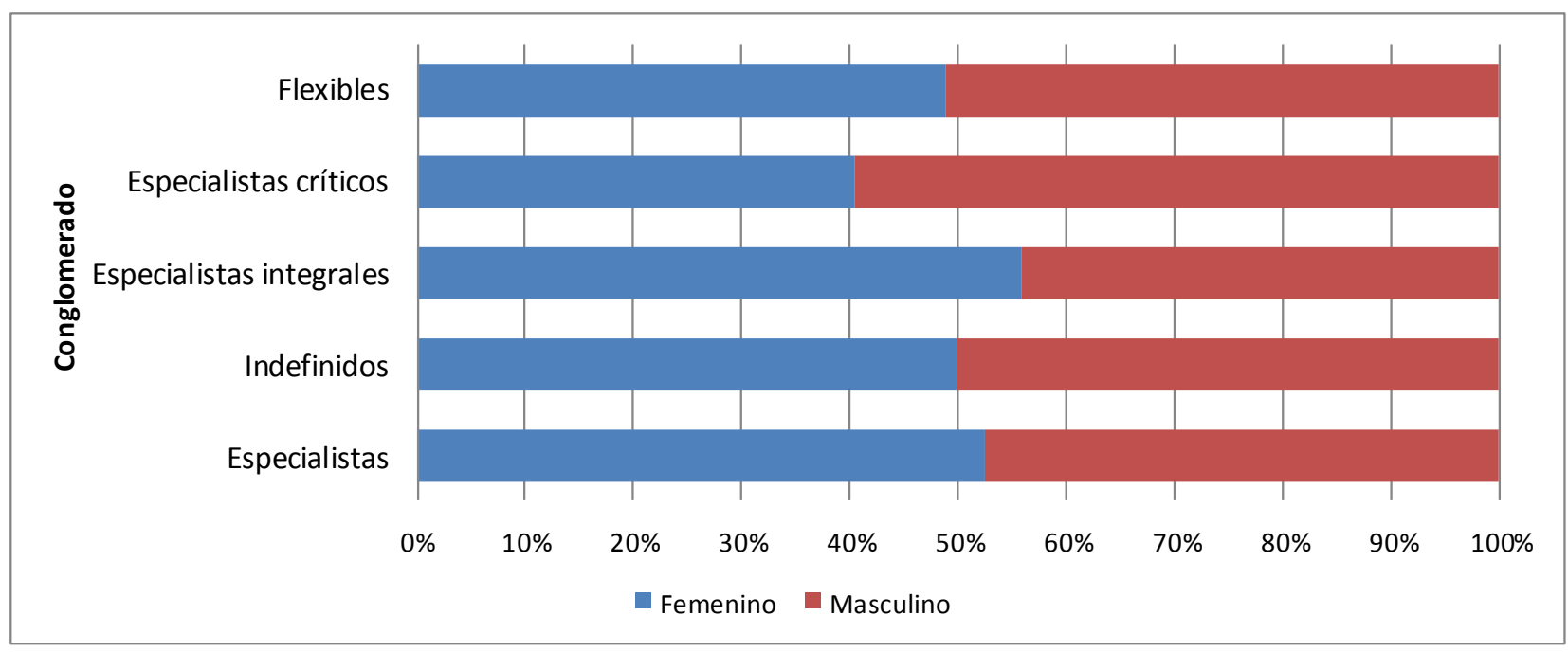

Fig. 8: Composición según sexo de conglomerados. 


\section{Conglomerado 1: "Especialistas"}

Este conglomerado agrupa poco más del $16 \%$ de los casos (Tabla 2 ) y representa lo que teóricamente se ha definido como un bajo nivel de flexibilidad. Los estudiantes que se encuentran dentro de este conglomerado podemos cualificarlos como de un perfil vocacional cerrado, es decir, se caracterizan por postular a carreras dentro de pocos dominios disciplinarios y en pocas instituciones de educación superior, a su vez evalúan de manera positiva la organización, interacción y articulación de las distintas áreas de conocimiento en el colegio y creen que la educación recibida durante enseñanza básica y media fue significativa, necesaria y útil. Finalmente otro rasgo que define a estos estudiantes es que su construcción de imaginario de carrera universitaria tiende hacia la especialización profesional, es decir, esperan una carrera que se oriente a un campo específico del conocimiento.

Los perfiles clasificados bajo este grupo se asocian a lo que la literatura ha definido como con un alto nivel de decisión y en este caso se encuentran vinculados principalmente a la elección de carreras del área "Salud". Si bien los porcentajes de participación relativa de los distintos tipos de establecimientos son similares, existe una tendencia hacia encontrar mayor cantidad de estudiantes de establecimientos municipales. Sin embargo, al controlar esta relación por la variable sexo, encontramos que esta tendencia se invierte en los hombres, donde encontramos más hombres de establecimientos particular pagados, sobre todo de la elección de carreras del área "Ingeniería y tecnología".

Tanto a nivel general, como en el caso específico de los hombres del grupo, este perfil se encuentra fuertemente vinculado con la elección de carreras de difícil ingreso, donde quienes postulan a ellas deben concentrar sus esfuerzos en sortear las barreras de entrada, lo que pudiese estar condicionando su nivel de flexibilidad cognitiva.

Tabla 2. Estadígrafos descriptivos para Conglomerado "Especialistas"

\begin{tabular}{|c|c|c|c|c|}
\hline \multicolumn{2}{|c|}{} & INFLEXED & INEVORIACE & INICU \\
\hline \multicolumn{2}{|c|}{ N Válidos } & 198 & 198 & 198 \\
\hline \multicolumn{2}{|c|}{ Media } & 4,48 & 17,09 & 17,80 \\
\hline \multicolumn{2}{|c|}{ Desv. estándar } &, 643 & 3,810 & 3,997 \\
\hline \multirow{3}{*}{ Percentiles } & 25 & 4,00 & 15,00 & 14,00 \\
\cline { 2 - 5 } & 50 & 4,00 & 18,00 & 18,00 \\
\cline { 2 - 5 } & 75 & 5,00 & 20,00 & 20,00 \\
\hline
\end{tabular}

\section{Conglomerado 2: "Flexibles"}

Este conglomerado, que agrupa poco más del $25 \%$ de los casos en análisis, se corresponde con lo que teóricamente identificamos como flexibilidad cognitiva, presentando altos valores en los tres índices (Tabla 3). Respecto al primero (INFLEXED), los estudiantes que se encuentran aquí clasificados, se caracterizan por escoger carreras de diversos dominios disciplinares en múltiples instituciones. La evaluación de la organización, interacción y articulación de las distintas áreas de conocimiento en el colegio (INEVORIACE) se encuentra condicionado por el género y la dependencia administrativa del establecimiento, siendo mejor la evaluación de su experiencia escolar de las estudiantes de los establecimientos particulares pagados, situación que provoca que no se aprecie una tendencia específica del índice dentro de este conglomerado. Respecto a la construcción del imaginario de carrera (INICU), encontramos que los puntajes tienden hacia la preferencia de carreras inclinadas a la formación integral e interdisciplinaria.

Esta categoría representa un perfil vocacional que bajo las clasificaciones convencionales no es relevado como tal en tanto no se diferencia de los "indefinidos". La caracterización aquí enunciada, permite su distinción a partir de la incorporación de la evaluación y proyección de la educación en términos de la flexibilidad e integración de contenidos. Los integrantes de este grupo, teóricamente debiesen presentar trayectorias más exitosas bajo curriculum más flexibles, al contrario de lo que sucedería con el caso de los del primer grupo.

En este grupo resulta significativa la relación con la dependencia administrativa, donde tanto hombres como mujeres de establecimientos particular pagados representan una proporción mayor. Al controlar la relación de dependencia administrativa según sexo, encontramos que esta tendencia es mucho más marcada en el grupo de las mujeres de los establecimientos particulares, situación que se explica principalmente por las 
diferencias en torno al imaginario de carrera, siendo coherente con la hipótesis respecto al sentido del imaginario y valoración social de la educación por parte de las mujeres (Koch, Contreras y Oliva, 2014).

\section{Conglomerado 3: "Especialistas integrales"}

Este grupo incluye cerca del 14\% (Tabla 4) de los casos, los que se caracterizan por escoger carreras de solo un dominio disciplinar y en pocos establecimientos educacionales, lo que lleva a que presenten bajos puntajes de INFLEXED. En lo referente a la evaluación de su experiencia respecto de la articulación, organización e interacción entre las distintas áreas del conocimiento, presentan puntajes relativamente bajos, lo que indicaría que en general perciben como significativa y necesaria la educación recibida. La particularidad de los integrantes de este conglomerado, es que presentan un imaginario de carrera que tiende hacia la apertura y flexibilidad, es decir, estos estudiantes desearían estudiar una carrera con contenidos diversos, formación integral y tienen una expectativa de trabajo transdisciplinario, situación que los diferencia de los que hemos denominado como "Especialistas".

Tabla 3. Estadígrafos descriptivos para Conglomerado "Flexibles"

\begin{tabular}{|c|c|c|c|c|}
\hline \multicolumn{2}{|c|}{} & INFLEXED & INEVORIACE & INICU \\
\hline \multicolumn{2}{|c|}{ N Válidos } & 317 & 317 & 317 \\
\hline \multicolumn{2}{|c|}{ Media } & 8,94 & 24,04 & 29,77 \\
\hline \multicolumn{2}{|c|}{ Desv. estándar } & 2,160 & 4,368 & 5,890 \\
\hline \multirow{3}{*}{ Percentiles } & 25 & 7,00 & 21,00 & 26,00 \\
\cline { 2 - 5 } & 50 & 8,00 & 24,00 & 29,00 \\
\cline { 2 - 5 } & 75 & 10,00 & 26,00 & 33,00 \\
\hline
\end{tabular}

En este caso existe una relación tanto con la dependencia del establecimiento como el sexo, en el sentido de que se aprecian más casos de establecimientos particulares y particular subvencionados, además de un mayor número de mujeres. Situación que se explica principalmente por las concepciones diferenciales en términos del imaginario de carrera que presentan estos grupos.

Tal como sucedía en el conglomerado "flexibles", no existe un dominio disciplinar que prevalezca por sobre los demás, aunque en el nivel del tipo de carreras escogidas, destacan profesiones que no son dominantes en su campo, como son "Enfermería" o "Diseño", situación que podría asociarse efectivamente a un imaginario de flexibilidad en estas profesiones.

Tabla 4: Estadígrafos descriptivos para Conglomerado "Especialistas integrales"

\begin{tabular}{|c|c|c|c|c|}
\hline \multicolumn{2}{|c|}{} & INFLEXED & INEVORIACE & INICU \\
\hline \multicolumn{2}{|c|}{ N Válidos } & 179 & 179 & 179 \\
\hline \multicolumn{2}{|c|}{ Media } & 4,46 & 19,99 & 28,32 \\
\hline \multicolumn{2}{|c|}{ Desv. estándar } &, 655 & 2,542 & 4,255 \\
\hline \multirow{3}{*}{ Percentiles } & 25 & 4,00 & 18,00 & 25,00 \\
\cline { 2 - 5 } & 50 & 4,00 & 20,00 & 27,00 \\
\cline { 2 - 5 } & 75 & 5,00 & 22,00 & 31,00 \\
\hline
\end{tabular}

\section{Conglomerado 4: "Especialistas críticos"}

Los estudiantes que componen este conglomerado (poco más del 17\%) se caracterizan por realizar una postulación cerrada, esto quiere decir que, postulan a carreras que se distribuyen en pocos dominios disciplinares. La característica que diferencia a estos estudiantes de los "Especialistas" es la negativa evaluación que hacen de la organización, interacción y articulación de las distintas áreas de conocimiento en su experiencia escolar, obteniendo altos puntajes de INEVORIACE. Respecto al imaginario de carrera, existen diferencias importantes entre ellos, teniendo el INICU una variabilidad del $26 \%$, siendo la más alta de todos los grupos, no siendo una característica relevante de este grupo su imaginario de carrera (Tabla 5).

En este grupo, se re-especifica la relación a nivel general entre la valoración escolar y el sexo, donde consecuentemente este grupo se encuentra conformado principalmente por hombres de establecimientos 
municipales; los que en términos generales escogen carreras vinculadas al área de "Ingeniería y Tecnología", especialmente ingenierías, lo que podría suponer un condicionamiento asociado a los requisitos de ingreso similar a los del conglomerado "Especialistas".

\section{Conglomerado 5: "Indefinidos"}

Finalmente, los casos que hemos calificado como indefinidos (26\%), se refieren a estudiantes que presentan características ambivalentes, mientras que por una parte presentan altos puntajes de INFLEXED, lo que indica que realizan una postulación a carreras de diversos dominios en diversas instituciones; presentan bajos niveles en INEVORIAC e INICU (Tabla 6), lo que indica bajo nivel de crítica en términos retrospectivos y un imaginario de carrera tendiente más bien a la clausura disciplinar.

Este grupo corresponde a lo que la literatura identifica tradicionalmente como indecisión, en el sentido de que si bien realizan postulaciones a carreras de distintos dominios disciplinares, no existen evidencias que nos permitan indicar que esta postulación refleje flexibilidad cognitiva en tanto, al menos a nivel consciente, no hay indicios de flexibilidad ni a nivel retrospectivo ni proyectivo. Es interesante destacar que no existe mayor relación de la dependencia del establecimiento ni el sexo del estudiante en la composición de este conglomerado, sindicándose como un fenómeno transversal, lo que permite hipotetizar que la variable desigualdad en el acceso a la información no parece ser un elemento diferenciador para la decisión en el caso chileno.

Tabla 5. Estadígrafos descriptivos para Conglomerado "Especialistas críticos"

\begin{tabular}{|c|c|c|c|c|}
\hline \multicolumn{2}{|c|}{} & INFLEXED & INEVORIACE & INICU \\
\hline \multicolumn{2}{|c|}{ N Válidos } & 215 & 215 & 215 \\
\hline \multicolumn{2}{|c|}{ Media } & 4,73 & 27,95 & 25,28 \\
\hline \multicolumn{2}{|c|}{ Desv. estándar } & 1,173 & 3,191 & 6,577 \\
\hline \multirow{3}{*}{ Percentiles } & 25 & 4,00 & 25,00 & 21,00 \\
\cline { 2 - 5 } & 50 & 4,00 & 27,00 & 25,00 \\
\cline { 2 - 5 } & 75 & 5,00 & 30,00 & 30,00 \\
\hline
\end{tabular}

Tabla 6. Estadígrafos descriptivos para Conglomerado "Indefinidos"

\begin{tabular}{|c|c|c|c|c|}
\hline \multicolumn{2}{|c|}{} & INFLEXED & INEVORIACE & INICU \\
\hline \multicolumn{2}{|c|}{ N Válidos } & 325 & 325 & 325 \\
\hline \multicolumn{2}{|c|}{ Media } & 9,27 & 17,91 & 19,26 \\
\hline \multicolumn{2}{|c|}{ Desv. estándar } & 2,412 & 3,732 & 4,823 \\
\hline \multirow{3}{*}{ Percentiles } & 25 & 8,00 & 16,00 & 16,00 \\
\cline { 2 - 5 } & 50 & 8,00 & 18,00 & 19,00 \\
\cline { 2 - 5 } & 75 & 11,00 & 20,00 & 23,00 \\
\hline
\end{tabular}

\section{DISCUSIÓN FINAL}

El actual modelo de financiamiento de la educación superior en Chile estaría influyendo en los perfiles vocacionales, condicionando la flexibilidad. Este factor estructural, potenciaría ciertos elementos culturales asociados al capital cultural de las familias que explican en parte las decisiones. Los resultados apuntan a evidenciar las importantes influencias socioestructurales que convergen en la construcción vocacional. Asimismo, ello contribuiría a configurar un rango de distinciones en torno a la noción de transdisciplina no transversal a los procesos educativos en el país.

Finalmente, destacamos tanto a través de los datos presentados, como a partir de otros productos de esta línea de investigación (Koch, Contreras y Oliva, 2014), que la inespecificidad en la elección, que tradicionalmente ha sido identificada como inmadurez o indecisión, desde la psicología educacional y la literatura vocacionalista, puede ser interpretada desde otra perspectiva, identificando estos enfoques como mecanismos de protección de las barreras disciplinarias. En este sentido, los resultados de este estudio nos permiten ciertos fundamentos para plantear que no necesariamente todos los casos de indefinición de un 
campo específico del saber en la elección, implicarían necesariamente un problema del estudiante o de los mecanismos de orientación vocacional, sino que pudiese deberse a que las universidades a través de su estructura parcelada en Facultades y Departamentos, no están siendo capaces de acoger perfiles vocacionales disonantes a esos patrones de organización del conocimiento.

En este sentido, la actual clausura identificada en el sistema universitario chileno, estaría afectando principalmente a aquellos estudiantes que presentan un perfil vocacional que tiende hacia la flexibilidad, pudiendo provocar problemas de deserción de sus respectivas carreras, ya sea para cambiar de área de estudio o para abandonar definitivamente el sistema. El relevar variables como las aquí expresadas, señala la importancia de que las universidades conozcan el perfil sus nuevos estudiantes, no solo desde una perspectiva socioeducativa, sino que también incorporando variables más complejas que permitan entregar una oferta acorde a los requerimientos de sus estudiantes.

Complementariamente, la predominancia de enfoques reduccionistas y la falta de principios organizativos complejos e integrativos en la configuración predominantemente asignaturista del curriculum escolar, tendrían importantes influencias en las dinámicas de postulación a las universidades por parte de estudiantes de cuarto medio en el país, generando hologramática y recursivamente modelos curriculares con bajos grados de complejidad, flexibilidad e integración inter-transdisciplinaria en el contexto universitario. Resultados como los reportados levantan ciertos perfiles y regularidades estadísticas en los procesos decisionales de jóvenes secundarios, los que si bien permiten levantar conclusiones preliminares, necesariamente deberán ser triangulados con metodologías cualitativas complementarias orientadas a cartografiar los procesos interpretativos en torno a la noción de inter/transdisciplina y su prospectiva en el despliegue de procesos formativos en universidades del país.

\section{CONCLUSIONES}

Los resultados de esta investigación fijan la mirada sobre aspectos no considerados tradicionalmente en el estudio de los procesos decisionales de postulación a carreras, atendiendo a los grados de flexibilidad vocacional y disciplinar observados en estudiantes secundarios desde la prospectiva del desarrollo transdisciplinario en universidades chilenas.

A partir de los datos, podemos concluir que en general la muestra tiene bajos niveles de flexibilidad en la postulación simulada y esta diferencia, si bien es más marcada en el caso de los hombres, no es relevante estadísticamente. Situación distinta sucede cuando se asocia a la dependencia del establecimiento del estudiante, el que podemos considerar un indicador de la condición socioeconómica, factor que si estaría influyendo significativamente en la configuración de los sujetos en estudio.

\section{AGRADECIMIENTOS}

La presente investigación recoge parte de los resultados obtenidos en el proyecto Fondecyt $\mathrm{N}^{\circ} 1120863$; "Posibilidades y condicionantes auto-eco-organizacionales para el desarrollo de procesos intertransdisciplinares en educación terciaria: Un programa complejo de investigación, desarrollo y divulgación”.

\section{REFERENCIAS}

Arvidsson, A. General Sentiment: how value and affect converge in the information economy. The Sociological Review, 59: 39-59 (2011)

Bourdieu, P y J.C Passeron. Los herederos: Ios estudiantes y la cultura. Siglo XXI, Buenos Aires, Argentina (2009)

Boyer R. y M. Freyssenet The productive models. The conditions of profitability, Palgrave, Londres, New York (2002)

Campion, M. y W. Renner The supposed demise of Fordism: Implications for distance education and higher education. Distance Education, 13(1), pp. 7-28 (1992)

Centro de Microdatos Universidad de Chile. Causas de deserción universitaria. Departamento de Economía. Universidad de Chile. Santiago. Chile (2008)

CNED. Instituciones de Educación Superior. (En línea) (2013) http://www.cned.cl/public/secciones/Seccion Educacion Superior/instituciones_educacion_superior.aspx Acceso: 20 de octubre de 2014

Coriat B., Trabajo y organización en la empresa japonesa. Madrid: Siglo XXI. (1992) 
CSE Deserción en la educación superior en Chile. (En línea) (2012) http://www.mineduc.cl/usuarios/ bmineduc/doc/201209281737360.EVIDENCIASCEM9.pdf Acceso: 20 de octubre del 2014.

Durkheim, E. The división of labour in Society, Free Press, New York, USA (2012)

Freyssenet M. Reflexión teórica sobre el concepto de "nuevo Modelo productivo" y perspectivas europeas en Crisis y empleo en Europa: Hacia un nuevo modelo productivo. El mundo que queremos. Andalucía, España (2011)

Gaete M. y R. Morales Articulación del sistema de educación superior en Chile: posibilidades, tensiones y desafíos. Calidad de la educación 25. pp 51-89. (2011)

Gamboa, V. Paixão, M. y S. Neves. Vocational profiles and internship quality among Portuguese VET students. International Journal for Educational and Vocational Guidance. 14(2), 221-244 (2014)

Gati, I., M. Krausz y S. Osipow. A taxonomy of difficulties in career decision- making. Journal of Counseling Psychology, 43, 510-526. (1996)

Germeijs, V. y P. De Boeck Career indecision: Three factors from decision theory. Journal of Vocational Behavior 62. Pp.1-25. (2003)

Jimenez D. La redistribution ESIC, Madrid, España (2009)

Kelly, K. y W. Lee Mapping the domain of career decision problems. Journal of Vocational Behavior, 61, 302326. (2002)

Koch, T. Contreras, T. y I. Oliva. Una carrera desigual. Sobre la "decisión" de (que) estudiar en la educación superior en Chile en En torno al trabajo sociológico. Reflexiones desde Playa Ancha. Universidad de Playa Ancha. Valparaíso. Chile (2014)

Larrain, C. Y S. Zurita The new student Ioan in Chile's Higher Education. Higher Education. 55 683-702. (2008)

Mehralizadeh, Y., E. Salehi y S. Marashi Higher education and the debate on key/generic skills, Canadian Journal of University Continuing Education, 34(1) pp.95-117. (2008)

Max-Neef, M. Fundamentos De La Transdisciplinaridad. Universidad Austral. Valdivia, Chile (2004)

Mc Whirter E.H y B. Mc Whirter Critical Perspectives on Adolescent Vocational Guidance in Chile. Journal of Career Development. 39(4) 386-404 (2012)

OCDE y BM La Educación Superior en Chile. (En línea) (2009) http://www.oecd-ilibrary.org/education/laeducacion-superior-en-chile_9789264054189-es;jsessionid=25wu5g4fg2dm4.x-oecd-live-01 Acceso: 21 de octubre de 2014

OCDE. Frascati Manual: Proposed Standard Practice for Surveys on Research and Experimental Development, 6th edition. (En línea) (2002)

http://www.oecd.org/science/inno/frascatimanualproposedstandardpracticeforsurveysonresearchandexperim entaldevelopment6thedition.htm Acceso: 20 de octubre 2014

OCDE y BM, El aseguramiento de la calidad en la educación superior en Chile. (2013) http://www.mineduc.cl/usuarios/1234/doc/201310151024490.2012\%20OCDEAseguramiento\%20Calidad.pdf Acceso: 20 de octubre de 2014.

Oliva, I., T. Koch y J. Quintero, Inter/transdisciplinariedad y universidad: Patrones de desplazamiento e interactividad disciplinar. Magis. Revista Internacional de Investigación en Educación. 6(13) pp.99-112. (2014)

PNUD Desarrollo humano en Chile. Bienestar subjetivo: el desafío de repensar el desarrollo. (En línea) 2012 http://www.desarrollohumano.cl/informe-2012/2012.zip Acceso: 20 de octubre de 2014

Salinas, D. y P. Fraser Educational Opportunity and Contentious Politics: The 2011 Chilean Student Movement. Berkley Review of Education. 3(1) 17-47 (2012)

Santos, P.J., J.A Ferreira y C.M Gonçalves Indecisiveness and career indecision: A test of a theoretical model. Journal of Vocational Behavior, 85(1), 106-114 (2014)

Smith A. La riqueza de las naciones. Alianza, España (2011)

Thomas D. y Menz F. The Vocational Assessment Protocol: Development and Validation. University of Wisconsin-Stout (1996)

Valenzuela, J., C. Bellei y D. De los Ríos. Socioeconomic school segregation in a market-oriented educational system. The case of Chile. Journal of Education Policy 29(2) (2014) 